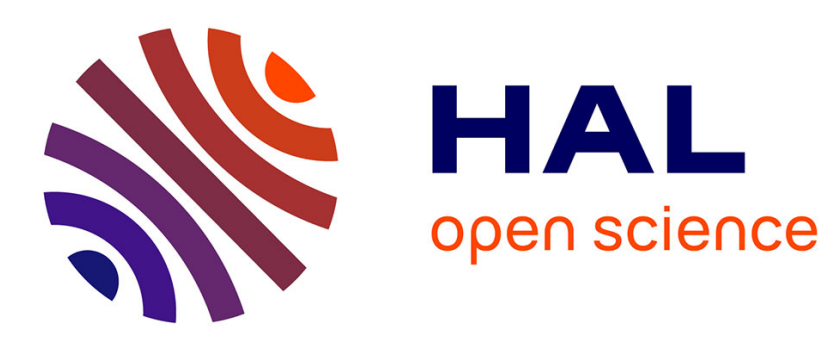

\title{
RELEVER LA FRANCE DANS LES APRÈS-GUERRES : RECONSTRUCTION OU RÉAMÉNAGEMENT?
}

Bertrand Vayssière

\section{- To cite this version:}

Bertrand Vayssière. RELEVER LA FRANCE DANS LES APRÈS-GUERRES: RECONSTRUCTION OU RÉAMÉNAGEMENT?. Guerres mondiales et conflits contemporains, 2009, 10 (236), pp.45-60. hal-00748603

\section{HAL Id: hal-00748603 \\ https://hal.science/hal-00748603}

Submitted on 5 Nov 2012

HAL is a multi-disciplinary open access archive for the deposit and dissemination of scientific research documents, whether they are published or not. The documents may come from teaching and research institutions in France or abroad, or from public or private research centers.
L'archive ouverte pluridisciplinaire HAL, est destinée au dépôt et à la diffusion de documents scientifiques de niveau recherche, publiés ou non, émanant des établissements d'enseignement et de recherche français ou étrangers, des laboratoires publics ou privés. 


\section{RELEVER LA FRANCE DANS LES APRÈS-GUERRES : RECONSTRUCTION OU RÉAMÉNAGEMENT ?}

Les guerres du XXe siècle ont provoqué des destructions à une échelle inconnue des autres périodes. Il serait vain de vouloir séparer en ce sens la Première Guerre mondiale, marquée par les épiques champs de bataille que sont Verdun, la Somme ou le Chemin des Dames, et la Seconde Guerre mondiale, caractérisée par les dommages faits aux civils et par la systématisation d'un génocide. Trois facteurs peuvent expliquer la montée en puissance destructive qu'orchestrent ces deux guerres, imitées par d'autres en ce «féroce XXe siècle $»^{1}$ : la « levée en masse » assure la participation du plus grand nombre à la politique, d'où découle l'importance de la propagande en tant que multiplicateur de volonté et de haine ; la peur rétrospective des «guerres populaires » qui ont agité le XIXe siècle, et qui accentue la «barbarisation» des affrontements, et ce dès $1914^{2}$; le processus de diabolisation de l'ennemi, assimilé au traître qui pervertit «l'harmonie » nationale, dont les effets se font ressentir là aussi dès la Première Guerre mondiale (recensement de 1916 des Juifs servant dans l'armée allemande qui participe de cette démonologie de l'ennemi intérieur; expropriation autoritaire de la valeur du travail qui commence avec le «communisme de guerre »; tentatives de déportation des Belges et des Polonais en octobre 1916). Au cours des deux conflits mondiaux, tous les engagements pris ont été violés : navires de commerce torpillés, gaz de combat employés, villes ouvertes bombardées, otages fusillés, civils massacrés. Le pillage n'a jamais été porté à un tel niveau d'aboutissement : classé par Aristote comme une industrie, au même titre que l'agriculture, admis par les juristes médiévaux et des Temps modernes comme «droit au butin » (jus praedarum), celui-ci se modernise jusqu'à devenir officiel, d'Etat à Etat : émission d'une monnaie spéciale, fixation de taux de change arbitraires, exportations obligatoires, mainmise sur les rouages de distribution. Dans les faits, la «brutalisation ${ }^{3}$ se traduit par des destructions inédites, résultats d'une technologie guerrière sans précédent (la «surprise technique » telle que la définit Raymond Aron) et d'une orchestration et d'une instrumentalisation de la violence qui atteint son comble.

C'est bien la raison pour laquelle les «phases de reconstruction» prennent alors une dimension si importante, au vu des ravages causés : les populations civiles, désormais parties prenantes de ces guerres totales, restent alors prisonnières des très nombreux décombres qui perdurent bien au-delà de l'armistice ou de la reddition sans conditions, traces apocalyptiques de la guerre qui perturbent leur quotidien (pénuries de tous ordres, ravitaillement plus ou moins prolongés, immeubles détruits ou branlants, toits effondrés, etc...). Ce sont ces victimes, mal logés ou sans abris, qui se tournent alors vers l'Etat pour qu'il améliore les conditions de vie, par l'intermédiaire des terrassiers, des entrepreneurs, des travaux publics : après les guerres, tout n'est pas pour le mieux, mais oui, le bâtiment va...

Penser la reconstruction, c'est aussi voir la façon dont les sociétés jugent la guerre et ses effets, et les conséquences au point de vue législatif. A ce propos, on peut constater, avec la définition progressive du «sinistré », l'idée généralisée que les pouvoirs publics ont pour devoir de considérer le risque de guerre au nom de la solidarité nationale, ce qui entraîne une évolution du droit dont tous les régimes placés face au problème de la reconstruction doivent tenir compte : l'indemnisation des dommages de guerre a tout autant été envisagée par la IIIe République (loi du 17 avril 1919), par le régime de Vichy (loi du 11 octobre 1940) que par la

\footnotetext{
${ }^{1}$ Robert Conquest, Le féroce XXe siècle : réflexions sur les ravages des idéologies, Paris, Ed. des Syrtes, 2001.

2 John Horne et Alan Kramer, 1914. Les atrocités allemandes, Paris, Tallandier, 2005.

${ }^{3}$ George L. Mosse, De la Grande guerre au totalitarisme. La brutalisation des sociétés européennes, Paris, Hachette-Littératures, 2003.
} 
IVe République naissante (28 octobre 1946), faisant du sinistré une figure incontournable des après-guerres et un objet nouveau de la compétition politique ${ }^{4}$.

Aujourd'hui, les historiens ne considèrent plus les conflits en eux-mêmes, mais comprennent l'intérêt des périodes qui les suivent, où tout est à rebâtir ${ }^{5}$. Cela revient à s'interroger sur le terme de «reconstruction»: est-il synonyme d'après-guerre? Désigne-t-il un phénomène économique et social? Doit-on s'en tenir aux problèmes architecturaux posés par les destructions ? Peut-on observer à l'échelle urbaine un continuum entre les reconstructions de la Première puis de la Seconde Guerre mondiales ? Nous tenterons de voir, à travers l'exemple français, hélas riche en exemples dans la première moitié du siècle $(1918,1945$ et, dans une certaine mesure au point de vue des réalisations administratives et juridiques, 1940), si reconstruction rime bien avec renouveau.

\section{I- $\quad$ Destructions et déblaiements.}

1) Une conséquence directe de la guerre totale.

L'après-guerre est, par définition, une période de remise en ordre, mais cette remise en ordre dépend du contexte politique, économique et social dans lequel on tente de l'effectuer, et plus prosaïquement de l'étendue des dégâts et des moyens dont on dispose pour les effacer. Si l'on prend en compte les deux après-guerres qui concernent la France, le constat est que les destructions, des points de vue quantitatif et qualitatif, sont très différentes en 1918 et en 1945 :

-660.000 bâtiments d'habitation sont touchés à l'issue du premier conflit mondial contre 1.900.000 après le second (soit $18 \%$ du capital immobilier); dans le premier cas, ce sont les zones rurales d'un seul tenant, concentrées sur 13 départements, qui ont le plus souffert, alors qu'en 1945, ce sont des villes entières qui ont été « coventrysées » (Le Havre, Caen, Saint-Nazaire, etc...) : 1.851 communes de 64 départements furent ainsi ravagées entre 1939 et 1945 (à comparer aux 80\% de la surface bâtie des principales villes en Allemagne «volatilisée » par les flottes alliées depuis des bases lointaines). Le paysage lunaire qui en découle fait aujourd'hui partie de notre patrimoine mental, sans lequel on ne peut imaginer qu'une guerre a eu lieu ; ces images terribles font d'autant plus partie de notre passé et ont tant marqué nos sensibilités que certaines d'entre elles ont été utilisées afin de susciter la compassion et l'aide à la reconstruction de puissances étrangères, en particulier aux EtatsUnis.

-20.000 établissements industriels ont été détruits en 1914-1918 contre 120.000 en 1940-1945 ; en 1918, ces destructions sont cantonnées à la région Nord-Est, théâtre principal des opérations; en 1945, elles sont beaucoup plus diffuses et se prolongent jusqu'aux principales infrastructures, volontairement endommagées à l'extrême fin du conflit, par un camp ou l'autre, le plus souvent pour des raisons stratégiques (4.000 ponts fluviaux, 7.500 ponts routiers, 115 gares, 1.900 ouvrages d'art, $22.000 \mathrm{~km}$ voies de chemin de fer).

-200.000 exploitations agricoles sont atteintes en 1918 (ce qui représente 3 millions d'hectares), 250.000 en 1945, détruites pour les mêmes raisons que précédemment ${ }^{6}$.

\footnotetext{
4 Eric Duhamel, «Les sinistrés, objet et enjeu politique », in Dominique Barjot, Rémi Baudouï, Danièle Voldman (dir.), Les reconstructions en Europe (1945-1949), Ed. Complexe, Bruxelles, 1997, pp.233-246.

5 Antoine Picon (dir.), La ville et la guerre, Besançon, éditions de l'Imprimeur, 1996 ; Rainer Hudemann et François Walter (dir.), Villes et guerres mondiales en Europe au XXe siècle, Paris, L'Harmattan, 1997 ; de manière plus générale, Tony Judt, Après-Guerre. Une histoire de l'Europe depuis 1945, Paris, Armand Colin, 2007.

${ }^{6}$ Ces chiffres sont donnés dans la plaquette du ministère de la Reconstruction et de l'Urbanisme, $L a$ Reconstruction française, bilan d'activité, 1945-1962, et dans la revue Urbanisme, n45/48, 1956 ; pour les chiffres établis sur l'instant, se reporter à : Commission consultative des dommages et des réparations,
} 
La guerre totale signifie la recherche de la victoire à tout prix, par tous les moyens, ce qui place les espaces urbains au cœur des objectifs stratégiques. Ainsi que l'écrit Jean Bacon : «Plus personne n'est à l'abri. On a parfaitement le droit de bombarder les hôpitaux : ils remettent sur pied ceux que les armes ont mis hors de combat. On peut bombarder les maternités : elles donnent de futurs ennemis à la patrie. On peut bombarder les écoles : c'est là que sont éduqués les jeunes qui feront l'armée de demain. On peut bombarder les églises : les fidèles y trouvent un réconfort qui leur permet de poursuivre la lutte. Et saper les fondations d'un musée ou d'une bibliothèque, c'est saper le moral de l'adversaire en le coupant de tout son passé culturel $\gg^{7}$. A partir des cas encore isolés de Sébastopol bombardée ou d'Atlanta incendiée, jusqu'aux dégâts généralisés des villes du front en 14-18 puis des horreurs de Dresde et d'Hiroshima, la ville n'est plus à l'abri, d'autant plus que les nouvelles armes permettent de l'atteindre de très loin (que l'on pense à la Grosse Bertha qui envoya ses obus en mars 1918 depuis une distance de $100 \mathrm{~km}$ sur Paris, ou au premier raid des Gothas allemands sur Londres en mars 1917 à partir de la Belgique occupée). 1914-1918 signifie bien une coupure dans «l'art de la guerre », ce passage à l'engagement total qui amènera les dirigeants à envisager en même temps le conflit et la reconstruction : c'est la loi du 25 août 1915 qui prescrit, pour la Belgique future, un plan général de reconstruction ; en France, ce sont les différents offices et services ad hoc créés au fur et à mesure de la guerre qui fusionnent le 16 novembre 1917, lors de la création du ministère du Blocus et des Régions libérées. Les solutions envisagées restent de toute manière d'ordre national : Jean Monnet, qui avait proposé et animé pendant le conflit une organisation des efforts maritimes et économiques de l'Angleterre et de la France, aurait voulu que ces conseils et comités soient maintenus après la guerre pour assurer une reconstruction harmonieuse de l'Europe, sans parvenir à imposer ses idées ${ }^{8}$.

L'idée de s'attaquer aux non-combattants, dans un combat déréalisé où les destructions se font à distance, par voie aérienne ou par obus interposés, annonce le passage à une guerre totale, où la ville, en tant qu'unité de production mais également centre de vie culturelle et sociale de l'ennemi, prend toute son importance stratégique : selon le général Giulio Douhet, directeur de l'aéronautique militaire italienne de 1912 à 1915, bombarder, et éventuellement détruire cet espace peut accélérer la fin de la guerre car, tout en endommageant des centres vitaux, on use d'une arme psychologique de premier ordre, pensée qui fera de nombreux émules ${ }^{9}$. De toute manière, l'insertion des villes dans les plans des différents états-majors amènent certains adeptes du «bombardement stratégique » à élaborer dans le même temps des solutions pour préserver les espaces urbains des destructions futures ${ }^{10}$. Si l'on retient le seul exemple des destructions urbaines, il est évident que les dégâts de 1945 sont à l'image d'une guerre qui a gagné en intensité, puisqu'elle ne peut avoir qu'une fin apocalyptique. Elle est à l'image de celle que Hitler prédisait à son peuple alors que la défaite paraissait inéluctable, et au moment même où son entourage, en particulier Albert Speer, lui suggérait de changer de tactique au vu

Evaluation des dommages subis par la France du fait de la guerre et de l'occupation ennemie, «Chiffres approximatifs et provisoires », automne 1945 ; voir tableau en annexe I.

7 Jean Bacon, Les saigneurs de guerre, Paris, Phébus, 2003 (rééd.), p.57.

${ }^{8}$ Jean Monnet, Mémoires, Paris, Fayard, 1976, pp.85-87.

${ }^{9}$ Giulio Douhet, Il Domino dell'Aria (La maîtrise de l'air), sorti en Italie en 1921 ; voir Dominique David, «Douhet ou le Dernier Imaginaire », Stratégique, janvier 1991, p.221. L'officier américain William Mitchell, l'un des pères de l'US Air Force, met en avant des considérations plus « humanitaires » : détruire massivement permet immanquablement d'abréger les souffrances de la guerre (Winged Defence, 1925, Skyways, 1930). Churchill comme Goering, et tant d'autres, reprendront cette antienne (atteindre le moral des populations ennemies de manière à les détacher de leur gouvernement), qu'une observation des faits pratiques de la guerre semble totalement infirmer.

${ }^{10}$ Exemple de Paul Vauthier, Le Danger aérien et l'Avenir du pays, Paris, Berger-Levrault, 1930, étudié entre autres cas par Efi Markou, «Militaires et urbanistes durant les années trente », Les Annales de la recherche urbaine, ${ }^{\circ} 91$, «Villes et guerres », décembre 2001, pp.18-26. 
de l'avance et des destructions ennemies : «Que veut dire tout cela, Speer ! Cela me fait rire, voilà tout. L'avance de l'ennemi est précisément notre chance. Les hommes ne se battent avec fanatisme que quand la guerre arrive à leur porte. Ils sont ainsi, que voulez-vous... Aucune ville ne sera livrée à l'ennemi avant d'avoir été réduite à un tas de décombres ${ }^{11}$. Conception archaïque, en pleine guerre moderne, d'une victoire qui ne s'arracherait pas grâce à la supériorité technique! Celle-ci est bien alors du côté des Anglo-saxons, qui bombardent jour et nuit les villes allemandes, mêlant objectifs stratégiques et (parfois involontairement) psychologiques, souvent en dépit du bon sens : l'application de la stratégie du «tapis de bombes » (area bombing) mise en œuvre par le chef de la flotte de bombardement stratégique de la Royal Air Force, Arthur Harris, avec l'assentiment de Winston Churchill, entama à peine le potentiel industriel de l'Allemagne nazie ; parallèlement, la population, mue par un besoin de protection mais également choquée par l'absurdité des massacres, eut tendance à se rapprocher du régime ${ }^{12}$. Précisons que cette façon d'envisager la guerre n'est pas une «création » de la Seconde Guerre mondiale : la première victime civile de bombardement «morte pour la patrie » remonte au mois de février 1918 à Paris ${ }^{13}$.

\section{2) Les permanences et les diversités dans l'action de l'Etat.}

Quelles ont été les réponses des pouvoirs publics face aux désastres des différentes guerres qui ravagèrent le sol national ? Dans les faits, elles sont proportionnées à l'étendue de ces destructions et aux moyens, pratiques et psychologiques, dont le pouvoir dispose. On constate donc bien une évolution d'un après-guerre à l'autre. En 1919, le gouvernement d'union nationale pensait la reconstruction en termes d'économie générale : le bâtiment était mis sur le même plan que l'industrie sidérurgique ou ferroviaire. De plus, si les régions du Nord et de l'Est étaient dévastées, une grande partie du territoire avait été épargnée. En 1940, le domaine urbain s'est dégagé des préoccupations globales du premier après-guerre. Mais dans une situation de paralysie et de pauvreté absolue, la réflexion a primé l'action; de même, on constate que, dans le domaine architectural, il n'y a pas d'unité mais au contraire dualisme entre une Délégation générale à l'équipement national d'une part, et un Commissariat à la Reconstruction d'autre part. En 1945, enfin, la création d'un ministère de la Reconstruction et de l'urbanisme (décret du 16 novembre 1944) consacre l'autonomisation du problème, avec comme premier titulaire Raoul Dautry ${ }^{14}$ (ministre de l'Armement en 1939!) ${ }^{15}$. Ainsi que l'écrit avec enthousiasme l'architecte Marcel Lods, ce ministère devrait être à même de réaliser enfin un plan général d'aménagement, permettant de «TOUT toucher, TOUT aborder, TOUT régir $»{ }^{16}$. Dans les faits, il a en effet des préoccupations diverses et variées, touchant à l'urbanisme, l'habitat et la construction, « y compris la lutte contre les taudis et les

\footnotetext{
${ }^{11}$ Cité in Joachim Fest, Albert Speer, le confident de Hitler, Paris, Perrin, 2001, p.221.

12 Joachim Fest, op. cit., pp.150-152. Pour le cas français, Claude d'Abzac-Epezy, «Les premiers bombardements alliés sur la France et leur utilisation politique », Revue historique des armées, n¹91, juin 1993, pp.73-84.

${ }^{13}$ Danièle Voldman, «Les populations civiles, enjeux du bombardement des villes (1914-1945) », in Stéphane Audoin-Rouzeau, Annette Becker, Christian Ingrao, Henry Rousso (dir.), La violence de guerre, 1914-1945, Bruxelles, Ed. Complexe, 2002, p.171.

${ }^{14}$ Les titulaires de ce portefeuille de 1944 à 1952 (année où tous les plans d'urbanisme sont étudiés et lancés) ont été dans l'ordre chronologique : Raoul Dautry (9 septembre 1944-20 janvier 1946), François Billoux (26 janvier 1946-28 novembre 1946), Jules Moch (16 décembre 1946-22 janvier 1947), Charles Tillon (22 janvier 1947-4 mai 1947), Jean Letourneau (9 mai 1947-22 octobre 1947), Jules Moch (22 octobre 1947-19 novembre 1947), René Coty (24 novembre 1947-7 septembre 1948), Eugène Claudius-Petit (11 septembre 1948-20 février 1952).

${ }^{15}$ A noter que la Belgique se dote d'un Commissariat du royaume aux grandes agglomérations le $1^{\text {er }}$ septembre 1941 et la Grande-Bretagne d'un Ministry of Town and Country Planning en 1944.

${ }^{16}$ Marcel Lods, «Un plan général d'équipement de la France », L'Architecture d'Aujourd'hui, nº $7 / 8$, septembreoctobre 1946.
} 
îlots insalubres », ainsi qu'à la réparation des dommages de guerre commis aux biens (ordonnance du 21 avril 1945). Pour ainsi faire, le ministère doit pouvoir compter sur un organigramme complet et spécialisé, fait de directions générales (de l'Urbanisme, des travaux), de commissariats (aux Dommages de guerre), d'inspections générales assurant la liaison entre le ministère et les délégués départementaux institués par l'arrêté du 11 mai 1945. Cependant, en matière de dommages ouvrant droit à l'indemnisation, on ne fait que reprendre la législation antérieure, parfois légèrement remaniée en fonction des circonstances; la principale différence est que l'Etat accepte d'établir le dialogue avec les victimes en encourageant la création d'associations de sinistrés (ordonnance n45-610 du 10 avril 1945).

Mais, au-delà de ces diversités, des tendances de longue durée se sont dégagées des analyses ponctuelles : l'importance du premier après-guerre comme exemple pour les reconstructions futures est essentielle. Grand référent au plan juridique et législatif, il sert à la définition des notions ultérieures de dommages, d'indemnité et de reconstitution du bien détruit (loi du 17 avril 1919 sur la réparation des dommages de guerre, complétée ensuite par 134 textes législatifs, qui ont accentué la complexité de la procédure et l'éparpillement des juridictions). C'est le 14 mars 1919 que la première loi organique française sur l'urbanisme est adoptée : la loi Cornudet subordonne le développement des villes importantes à un «plan d'aménagement, d'embellissement et d'extension », chaque commune se devant ainsi de préparer un plan conforme à des attentes plus globales. Cette disposition ne servait pour l'instant que de déclaration d'intention, dans la mesure où l'Etat, avec la loi Loucheur votée le 7 juillet 1928, privilégiait la quantité sur la qualité. Cette loi, accusée en son temps d'établir un programme individualiste et «propriétiste » ${ }^{17}$, prévoyait des subventions de l'Etat (1,4 milliards de francs par an jusqu'à la fin de 1933) pour la construction de 260.000 «maisons individuelles affectées aux logements des invalides de guerre, du travail, et aux familles nombreuses ». Ainsi donc, la loi encourageait l'extension de l'habitat individuel, qui allait imposer une marque anarchique à l'urbanisation de la banlieue parisienne dans les années 20. Néanmoins, elle signifiait l'importance de l'engagement de l'Etat dans un domaine où le secteur privé, découragé par l'importance des taux d'intérêt et le contrôle des loyers, était réticent. Le gel des loyers, décidé le 9 mars 1918, paralysait ainsi le marché de l'immobilier en freinant toute spéculation et en décourageant les propriétaires de restaurer leurs biens : en France (1939), $6 \%$ du revenu était consacré au loyer, contre 20\% en Allemagne, et 25\% aux Etats-Unis et en Grande-Bretagne. Ce constat ne signifie pas encore une mainmise globale de l'Etat sur l'économie, dans la mesure où celle-ci n'est pas possible dans tous les secteurs: le gouvernement français doit faire face au désir de certains milieux de revenir aux pratiques libérales d'avant-guerre, comme le montre l'échec du projet de nationalisation des chemins de fer introduit à la Chambre des députés en avril 1919 par Albert Thomas. Cela dit, il vérifie le fait que l'urbanisme est désormais pensé en tant que tel, dans ce que cela implique de généralisation et de rationalisation.

La question du logement social reste toujours aussi aiguë d'un après-guerre à l'autre, question ardue qui pose la question de la place de l'Etat dans la société, et qui renvoie automatiquement à l'autre grande question que l'on retrouve dans les deux périodes d'après-conflit, celle du décalage entre les recherches formelles des architectes (esthétisme et novation) et les conditions de la commande publique après les grandes destructions (urgence et rénovation). Tout concourre à une problématique des architectures qui, pour la reconstruction, met en avant la tabula rasa (naturelle ou militaire) aux dépens des continuités urbanistiques, jugées archaïsantes et reflets d'un temps obsolète qu'il s'agit de dépasser : la reconstruction serait ainsi une programmation volontaire de $l^{\prime}$ oubli ${ }^{18}$. Au-delà de l'aspect matériel, les projets

\footnotetext{
${ }^{17}$ Journal officiel : Chambre des Députés, débats, 3 juillet 1928, p.2226-2229. Pour la législation urbaine, voir tableau en annexe II.

${ }^{18}$ Voir à ce propos Marcel Smets, « Esquisse d'une théorie de la reconstruction », Urbanisme, 196, 1983.
} 
contenus dans les cartons de certains architectes modernistes seraient un des moyens naturels d'appliquer enfin une politique qui, au-delà de la réparation ou de la reconstitution des dégâts causés par la guerre, concernerait la société toute entière: pour le ministre de la Reconstruction et de l'Urbanisme Eugène Claudius-Petit (11 septembre 1948-20 février 1952), l'effort consiste d'abord à lutter contre une vétusté généralisée des bâtiments, témoin d'une situation d'entre-deux-guerres où l'on avait constaté un appauvrissement du parc de logements en France, avec un retard considérable sur nos voisins européens concernant les commodités (principalement l'adduction d'eau). Il s'agit de considérer, à côté des "sinistrés de la guerre », les «sinistrés de la vie », dont les habitations ne sont que des taudis au regard des exigences modernistes et hygiénistes, les seules à même de réaliser le projet de suture des traumatismes de la guerre que les aménageurs préparent, afin de matérialiser le reflux général de la mémoire et poser les bases d'une société meilleure.

\section{II- $\quad$ La guerre comme facteur de changement ?}

\section{1) L'exemple de Vichy et de la Résistance.}

Certes, la période qui suit immédiatement l'armistice de juin 1940 ne peut pas être considérée du point de vue formel comme un après-guerre, mais elle a été ressentie en tant que tel par une partie des contemporains. C'est pourquoi, en cette matière comme dans d'autres, la législation urbanistique, même théorique au niveau de son application en cette période d'occupation, servira amplement l'action de reconstruction entamée en 1945. On peut généraliser à l'urbanisme la réflexion lancée sur Vichy dans d'autres domaines, à savoir la recherche de rationalisation des procédures, des bases d'une véritable planification, à l'aide de principes corporatistes (création de l'Ordre des architectes par la loi du 31 décembre 1940, qui met en avant la nécessité d'un diplôme pour faire partie de la corporation, certes dans l'immédiat plus théorique que réelle ${ }^{19}$ ): de nombreuses pistes sont lancées en matière d'urbanisme, qui, dans le sillage de la débâcle, ressemblent plus à des incantations contre l'ancien système qu'à des propositions constructives, ne serait-ce que parce que les moyens, en temps de guerre, sont limités, voire inexistants ${ }^{20}$. On doit en particulier signaler la loi sur l'urbanisme (loi du 15 juin 1943) où, au-delà de la reconstruction elle-même, est envisagé en contact direct et étroit un effort d'aménagement et de planification urbaine. Mais ce débat n'est pas un produit typiquement « vichyste », puisqu'il ne fait que reprendre des discussions déjà lancées avant-guerre, qui tiennent enfin compte du phénomène d'extension et de croissance urbaine, et par là même interdisent de considérer la ville comme un système clos et figé (exemple des lois du 14 mai 1932 pour la région parisienne, élargi par le décret-loi du 25 juillet 1935 à toute la France, mais qui sont restées en grande partie lettre morte). Dans les faits, les projections vichystes privilégient cependant le passéisme sur la modernisation, à l'image du message global de la Révolution nationale, victime en la matière des volontés de l'occupant, qui avait fait montre de son irritation face aux audaces du Bauhaus ou aux tentatives d'habitat social des Siedlungen (mettons de côté les audaces architecturales qui ont surtout bâti la complicité stérile entre Hitler et Speer). La reconstruction, tout du moins son projet, est bel et bien le reflet d'un modèle de société, archaïsant et rural, comme le montre ce passage de la Charte de l'architecte reconstructeur de 1941: «Songez que depuis plus d'un demi-siècle, les petites villes, les campagnes, s'enlaidissent, les caractères régionaux s'effacent $[\ldots]$ N'hésitez pas à regarder les maisons traditionnelles des petites villes et des

\footnotetext{
${ }^{19}$ Rappelons que Le Corbusier ou Auguste Perret n'étaient alors même pas diplômés.

${ }^{20}$ Jean Vincent, La reconstruction des villes et des immeubles sinistrés après la guerre de 1940, Paris, Bischop et fils, 1943 ; publications des fascicules de L'Institut Technique du Bâtiment et des Travaux Publics, organisme rattaché à la Direction technique de la Reconstruction immobilière.
} 
villages : chefs-d'œuvre des artisans d'autrefois. Elles sont l'expression parfaite du genre de vie de nos ancêtres ${ }^{21}$. Ce ruralisme régionaliste et moraliste exclut toute approche sociologique ou économique du corps social, dans la mesure où il se fait le porte-parole d'une idéologie qui refuse de par son essence même les principes d'une société complexe.

Reconnaissons tout de même à la politique urbanistique de Vichy la volonté de prévoir l'aménagement rationnel des villes à l'occasion de la reconstruction, ce qui n'avait pas vraiment été fait en 1918 où celle-ci s'était limitée à la stricte réparation (indemnisation) des dommages de guerre sur le plan législatif. Cette préoccupation rencontre celle de certains architectes qui avaient réfléchi sur ce thème avant même la guerre, tels que Le Corbusier ${ }^{22}$. Il est vrai que, dans la Résistance, les débats sur la reconstruction sont encore assez limités, à Alger comme en France même: seuls quelques membres isolés, généralement cadres supérieurs, techniciens ou "grands commis de l'Etat », réfléchissent à un programme général (et assez technocratique) s'inspirant des thèses des CIAM (Congrès international d'architecture moderne), la Charte d'Athènes notamment ${ }^{23}$. Les partis restent encore marqués par une vision très partielle et idéologique des problèmes urbains, ainsi les communistes obnubilés par les problèmes immédiats des «mal-lotis ». De même, on ne trouve pas, parmi les positions des quelques organismes spécialisés créés pendant la guerre (Union nationale des ingénieurs et techniciens, Londres, 11 novembre 1942, Front national des Architectes, mars 1943) une véritable réflexion sur les principes architecturaux et urbanistiques à mettre en œuvre dans la reconstruction, le projet politique de «rebâtir la France » passant bien avant celui, plus économique et social, de « rebâtir les logements ». Néanmoins, on peut observer que certains des organismes gravitant autour du CNR ou du CFLN commencent à étudier la politique urbanistique de Vichy et à prendre en compte, vers la fin de la guerre, la nécessité d'encadrer par l'Etat l'effort de reconstruction, qui s'autonomise peu à peu : Jean Monnet, qui dirige le commissariat à l'Armement, à l'approvisionnement et à la reconstruction (créé le 3 juin 1943), n'a plus en charge, à partir du 14 octobre, que les deux derniers portefeuilles, la conviction étant qu'à partir de la Libération, ceux-ci devraient être confiés à un même ministère.

\section{2) Les politiques de reconstruction après la Libération.}

De manière générale, le problème de la reconstruction est rarement posé en terme de besoins en logements, même après 1945, où le premier réflexe consiste à « réparer », par le biais des indemnisations, ce qui a été détruit (logements ou patrimoine), parfois en se référant à des textes de lois datant de 1919 : on ne remet donc pas en cause le principe de la prise en charge intégrale par l'Etat du montant des dommages, accentué même par la loi du 28 octobre 1946 qui précise que cette réparation intégrale s'effectue dans le cadre du plan général d'équipement et de modernisation. Cependant, la réparation en question, qui doit se faire « sur place » et «à l'identique » (et donc souvent au prix fort) est plutôt tournée vers le passé et difficile à financer : selon les chiffres officiels, seulement $2 \%$ des 310.000 édifices totalement sinistrés étaient reconstruits à la date du $1^{\text {er }}$ juillet 1946, essentiellement les entreprises

\footnotetext{
${ }^{21}$ Charte de l'architecte reconstructeur, Imprimerie Nationale, Paris, 1941, p.10.

${ }^{22}$ Le Corbusier (Charles-Edouard Jeanneret), Les Quatre routes, Denoël-Gonthier, Paris, 1941 (rédigé en 1939) ; les «quatre routes » (terre, fer, air, eau) sont celles à partir desquelles l'aménagement du territoire doit être envisagé pour l'organisation des établissements humains, à partir d'un "programme », d'un «plan directeur » surveillé et appliqué par l'«harmonisateur», figure composite où se retrouvent les pouvoirs publics et les architectes; Le Corbusier expose au gouvernement de Vichy ses idées dans La Maison des hommes (en collaboration avec François de Pierrefeu), Plon, Paris, 1942.

${ }^{23}$ Maxime Blocq-Masquart (dirigeant de l'Organisation Civile et Militaire), Chroniques de la Résistance, Paris, Coréa, 1945.
} 
industrielles et les constructions officielles ${ }^{24}$. C'est seulement la loi du 18 juin 1948 qui annonce la mise en place d'une véritable politique de reconstruction et d'aménagement, par l'intermédiaire des associations syndicales et des sociétés coopératives de reconstruction : et encore, cette loi ne se réfère pas tant à la guerre mais plutôt à l'avenir, qui semble annoncer une crise du logement qu'il faut impérativement résorber. Elle permettra dans les faits d'appliquer certains principes préconisés par les «modernistes » (hygiène ${ }^{25}$, ensoleillement, «zoning », fonctionnalité de l'espace urbain) tout en privilégiant les méthodes industrielles et l'occupation d'espaces de plus en plus grands et d'un seul tenant. Il n'y a cependant pas rupture avec la conception traditionnelle de l'espace urbain, qui privilégie la construction d'îlots bordés de rues, ce qui dénote le manque d'un véritable plan directeur.

N'oublions pas les conditions d'après-guerre, qui obligent dans un premier temps à prendre en compte le désir des sinistrés de récupérer ce qu'ils avaient perdu, ce qui entraîne un travail hâtif au vu des besoins, qui par définition ne favorise pas l'effort de réflexion et de coordination. On aboutit ainsi à des «reconstructions historiques» (Saint-Malo), des « modernisations raisonnables » qui tentent de regrouper des services jusque-là dispersés en un même endroit tout en ne touchant pas à l'aspect des bâtiments et à la dispersion d'habitations qui restent le plus souvent individuelles (Saint-Lô, Rouen, Caen); rares sont les cas de «table rase », où sont conçus de véritables plans masse obéissant à une organisation générale, appliquant la notion moderne de trame à l'ensemble d'un cadre urbain, préconisant la mise en place de larges artères, l'alignement et l'homogénéisation des façades : ce cas s'applique dans certaines villes (Le Havre reconstruit par l'architecte Auguste Perret), mais la conception en est difficilement transposable dans la mesure où elle ne s'applique que dans les espaces urbains totalement détruits ${ }^{26}$. Qui plus est, on dénonce dans ces derniers projets l'aspect utopique, au regard des préoccupations plus terre-à-terre des habitants : l'exemple de Sarrebruck en Allemagne est à cet égard probant. Dans le cadre des relations spéciales qui devaient unir la France à ce Land, les autorités d'occupation, représentées par le commandant en chef des Forces françaises en Allemagne, le général Koenig, et le haut gouverneur militaire de la Sarre, Gilbert Grandval, imaginèrent une refonte complète des villes, dont certaines étaient effectivement entièrement détruites. Dans cette optique, un projet fut remis à GeorgesHenri Pingusson, architecte qui avait un temps collaboré avec Le Corbusier et participé à la préparation de l'Exposition universelle à Paris, en 1937. Le plan qu'il remît le 14 juillet 1947 comprenait le réaménagement complet des quartiers historiques de la capitale sarroise en créant des rangées linéaires de gratte-ciels à vitres reliés par un vaste axe routier d'est en ouest, une immense place centrale regroupant les principaux services (centre de vie publique avec la mairie, la direction des mines, l'ambassade de France) et tout un réseau permettant la réglementation des trafics (séparation selon les véhicules) ainsi qu'une circulation continue favorisant les contacts d'une zone à l'autre. Cependant, ces projets « futuristes » restèrent pour une large part sur le papier, rappelant précisément que les périodes d'après-guerres ne sont pas faites pour imaginer l'avenir mais d'abord pour panser les plaies : comme l'exprime un contemporain, «la question d'une annexion de la Sarre à la France sur le plan politique et économique ne suscita pas de débats incontrôlés. On pouvait définir l'atmosphère de manière suivante : qu' 'ils' fassent ce qu'ils veulent mais qu'on ne nous pose pas de questions. Par 'ils', on entendait à la fois les partis allemands, la France ou tous les Alliés. Cette attitude exprime la défaite totale telle qu'elle n'avait jamais été perçue à l'époque contemporaine.

\footnotetext{
${ }^{24}$ Résumé des chiffres officiels in La Maison française, « Où en est la reconstruction ? », 1946, 3, pp.13-20.

${ }^{25}$ Et encore, en 1954, 41,6\% des habitations en France n'étaient toujours pas munies d'eau courante et 73,4\% de W.C., Philippe Ariès et Georges Duby, Histoire de la vie privée en France ( $5^{\mathrm{e}}$ volume), Paris, Seuil, 1987, p.68.

${ }^{26}$ Patrick Dieudonné (dir.), Villes reconstruites. Du dessin au destin, 2 vol., Paris, L'Harmattan, 1994.
} 
Comment pouvait émerger, dans une telle grisaille, une volonté vraiment artistique ? $»^{27}$. On peut faire le même constat face aux projets avortés de reconstruction des villes de Saint-Dié ou de Mayence, où les suggestions de modernisation, s'appuyant sur l'étendue des destructions, sont malgré tout remises en cause par la population elle-même ${ }^{28}$. Pourtant, dans le premier cas, Le Corbusier estimait que «les conditions [étaient] exceptionnelles, [la] zone urbaine [étant] détruite de façon extrêmement favorable ${ }^{29}$.

Les plus novateurs des acteurs de la reconstruction se casseront les dents sur l'alternative reconstruction ou aménagement. Eugène Claudius-Petit, désireux de mettre en place une véritable politique moderne et audacieuse d'aménagement du territoire ne se limitant pas à une simple reconstruction à l'identique, est coiffé sur le poteau à la Libération par le très conventionnel Raoul Dautry, à la tête d'un ministère de la Reconstruction et de l'Urbanisme à peine épuré et d'un appareil législatif tout juste toiletté. L'innovation est alors dans l'opposition, et son message est clair : pour Claudius-Petit, c'est un plan directeur qu'il réclame à la tribune de l'Assemblée le 5 mars 1945, car « ou bien la France recommencera son passé, reconstituera un musée en toc [...] ou bien la France continuera sa tradition. Sa tradition? Elle est de tenir compte des événements, de tenir compte des possibilités nouvelles, de tenir compte des matières nouvelles, et de construire des villes jeunes, où l'homme sera réconcilié avec la nature ${ }^{30}$. C'est seulement le 11 septembre 1948 que Claudius-Petit devient ministre, alors que l'opinion semble plus ouverte à certaines idées novatrices en matière d'urbanisation $^{31}$. Il présente ses grands axes dans un livre «vert» intitulé Pour un plan national d'aménagement du territoire ${ }^{32}$, dans lequel sont exposées des idées relatives au rééquilibrage des activités en France, la volonté d'étudier les problèmes urbains à l'échelle globale de l'agglomération et de la région ainsi que celle de développer les transports en commun. Pour ce faire, Claudius-Petit décide de présenter une loi foncière permettant d'élargir le droit à l'expropriation de l'Etat mais, face aux réticences de certains (en particulier le Commissariat au Plan de Jean Monnet, qui estime que le ministère de la Reconstruction et de l'Urbanisme empiète sur ses prérogatives), cette loi, si nécessaire à la mise en place d'un plan directeur permettant à l'Etat d'avoir les coudées franches en matière d'urbanisation, sera repoussée à deux reprises par l'Assemblée nationale (2 novembre 1950 et 8 novembre 1951) puis discrètement enterrée. Finalement, la politique novatrice de Claudius-Petit ne parvient pas à s'implanter, se limitant à quelques réalisations, prestigieuses mais isolées (implantation en province d'usines prévues pour Paris comme Gillette à Annecy, Citroën à Rennes, Renault à Cléon). L'aménagement du territoire était repoussé sine die.

\footnotetext{
${ }^{27}$ Hans Groh, «Die Situation an der Saar », Aussaat, Heidelberg, 1947, p.126, cité dans Norbert Mengden, «L'urbanisme: utopie et réalité à l'exemple de Sarrebruck », in Francine-Dominique Liechtenhan, Europe 1946, entre le deuil et l'espoir, Ed. Complexe, Bruxelles, 1996, p.204 (note 22).

${ }^{28}$ Jean-Louis Cohen, Hartmut Frank et al., « Architettura dell'Occupazione : Francia e Germania 1940-1950 », Casabella, Milano, n567, Aprile 1990.

${ }^{29}$ Fondation Le Corbusier H (3) 18, Lettre de Le Corbusier à Raoul Dautry, 21 décembre 1945, p.1, cité dans Rémi Baudouï, «Imaginaire culturel et représentations des processus de reconstruction en Europe après 1945 », in Dominique Barjot, Rémi Baudouï, Danièle Voldman (dir.), op.cit., p.311. Sur Mayence, Rémi Baudouï, « La reconstruction française en Sarre, 1945-1950 », Vingtième Siècle, janvier-mars 1991, pp.57-65.

30 Journal officiel, Assemblée consultative, débat du 5 mars 1945, p.284-297. Voir Benoît Pouvreau, "La politique d'aménagement du territoire d'Eugène Claudius-Petit", Vingtième Siècle, n79, juillet-septembre 2003, pp.43-52.

${ }^{1}$ Voir en particulier l'écho du livre de Jean-François Gravier, Paris et le désert français, Paris, Le Portulan, 1947.

${ }^{32}$ Pour un plan national d'aménagement du territoire, ministère de la Reconstruction et de l'Urbanisme, Paris, février 1950, $28 \mathrm{p}$.
} 


\section{CONCLUSION.}

Ce n'est pas un hasard si les représentants les plus en pointe de l'urbanisme, Alfred-Donat Agache, Jean-Marcel Auburtin et Edouard Redont, font paraître leur livre, Comment reconstruire les cités détruites ${ }^{33}$ en 1915. Il s'agissait alors de faire aboutir les solutions modernistes proposées sans succès depuis la fin du XIXe siècle, et de mettre l'Etat devant ses responsabilités. Las, ces solutions réformatrices n'aboutissent pas, l'instabilité politique, les difficultés économiques et un certain état d'esprit individualiste repoussant l'application de plans d'ensemble propre à l'espace urbain. Dans le même temps, les militaires mettent au point des stratégies qui, elles, intègrent pleinement cet espace urbain comme cible prioritaire.

La reconstruction française à la suite de la Seconde Guerre mondiale n'a pas vraiment fait preuve d'audace architecturale et urbanistique, mais elle a mis en route une vision technicienne, inspirées des expositions organisées par le Ministère de la Reconstruction et de l'Urbanisme (des techniques américaines avec l'exposition de la National Housing Agency au Grand Palais $^{34}$, de la reconstruction,...) ou des missions aux Etats-Unis (délégation conduite par Claudius-Petit et Le Corbusier le 25 décembre 1945), qui toutes concluent à la nécessité de la rationalisation de la production (procédure d'agrément des matériaux et des procédés de construction). On constate que la période de reconstruction proprement dite (1945-1955) a plus servi de banc d'essai pour les années 60 (organisation des chantiers, préfabrication d'éléments de base) permettant le dépassement des méthodes artisanales d'avant-guerre, qu'elle n'a constitué une période architecturale à part entière, malgré de nombreux espoirs. La reconstruction a privilégié la «modernisation raisonnable », qui est le résultat d'un compromis entre ancien et nouveau, le nouveau n'entrant qu'à reculons, sous la forme la plus édulcorée possible ; la «maîtrise d'ouvrage » a été mise en avant, sans pour autant que l'on s'intéresse aux formes architecturales et urbanistiques. On est donc loin de la Révolution espérée par les modernistes des années 30, ce qui renvoie à l'échec subi par eux en leur temps dans les différents pays où ils se sont exprimés, mais aussi aux réalités sociales d'un pays qui n'a pas mécaniquement changé avec la fin de la guerre : on reconstruit le cadre d'une société qui reste avant tout le prolongement de celle d'avant-guerre.

Cependant, on ne peut que constater, dans le cas français, une tendance générale entre les deux conflits mondiaux: le renforcement des institutions administratives, des décisions législatives, des innovations techniques, des projets d'architecture et d'urbanisme à l'issue de chaque conflit. Ainsi, les plans d'aménagement établis entre 1919 et 1924 sont-ils perfectionnés en 1940, avant d'être repris en 1945 ; les associations de sinistrés, mis en place après le 11 novembre 1918, sont réactivées par Vichy, puis par la Quatrième République naissante. Chaque reconstruction s'est ainsi nourrie de la précédente, laissant de côté toute imagination créative, dans une sorte de «pédagogie du malheur » où le passé et l'avenir ont tous deux une place ambiguë. Peut-être cet état de fait permet-il de rappeler que destruction et reconstruction sont les deux facettes d'un jeu à somme nulle, dans lequel la nostalgie et l'appel au progrès n'arrivent pas à se départager ?

Bertrand Vayssière, Maître de conférences à l'Université Toulouse II- Le Mirail

\footnotetext{
${ }^{33}$ Donat-Alfred Agache, Marcel Auburtin, Edouard Redont, Comment reconstruire nos cités détruites. Notions d'urbanisme s'appliquant aux villes, bourgs et villages, Paris, Armand Colin, 1915.

${ }^{34}$ Anonyme, «Les techniques américaines doivent inspirer la construction française », La Maison française, 1946, 36, pp.50-69.
} 


\section{I - NATURE DES DOMMAGES :}

\begin{tabular}{|c|c|c|c|}
\hline \multicolumn{1}{|c|}{ Rype de bâtiments } & Détruits ou irréparables & $\begin{array}{c}\text { Valeur 1939 (en millions } \\
\text { de francs) }\end{array}$ & $\begin{array}{c}\text { Valeur 1945 (en millions } \\
\text { de francs) }\end{array}$ \\
\hline $\begin{array}{l}\text { Bâtiments à usage } \\
\text { principal d'habitation } \\
\text { (maisons, immeubles)..... }\end{array}$ & 751000 & 267600 & 909840 \\
\hline $\begin{array}{l}\text { Exploitations agricoles... } \\
\text { fndustriels ............... }\end{array}$ & 250000 & 40700 & 138380 \\
\hline
\end{tabular}

II- LOGEMENTS D'HABITATION $^{35}$

\begin{tabular}{|c|c|c|c|c|}
\hline \multicolumn{5}{|c|}{ Total des logements reconstruits : depuis la Libération jusqu'au 30 septembre 1950} \\
\hline \multirow[b]{2}{*}{ RECONSTRUCTIONS FAITES PAR L'ETAT : } & \multicolumn{2}{|c|}{$\begin{array}{l}\text { Logements } \\
\text { terminés }\end{array}$} & \multicolumn{2}{|c|}{$\begin{array}{l}\text { Logements en } \\
\text { cours de } \\
\text { construction }\end{array}$} \\
\hline & \multirow[b]{2}{*}{16085} & $\%$ & \multirow{2}{*}{4310} & \multirow{2}{*}{$\%$} \\
\hline Ministère de la Reconstruction et de & & 29 & & \\
\hline 8 & 140 & 1,1 & 25 & 0,1 \\
\hline & 2455 & 4,5 & 90 & 0,1 \\
\hline $\begin{array}{r}\text { Secteurs nationalisés }(\text { SNCF par exemple }): \ldots \ldots \ldots \ldots \ldots \ldots \ldots \\
\text { TOTAL: }\end{array}$ & 18680 & 34,6 & 4425 & 6,7 \\
\hline $\begin{array}{c}\text { SUR INDEMNITES DE DOMMAGES DE GUERRE OU PAR } \\
\text { PREFINANCEMENT SUR COMPTE SPECIAL }\end{array}$ & & $\%$ & & $\%$ \\
\hline $\begin{array}{l}\text { Par les groupements de sinistrés (associations syndicales, coopératives } \\
\text { et mandataires communs) ......................... }\end{array}$ & 6160 & 11,4 & 30075 & 45,5 \\
\hline Par les sinistrés (reconstruction individuelle) ..................... & 29235 & 54 & 31600 & 47,8 \\
\hline TOTAL & 35395 & 65,4 & 61675 & 93,3 \\
\hline TOTAL $\ldots \ldots \ldots \ldots \ldots \ldots \ldots \ldots \ldots \ldots \ldots \ldots \ldots \ldots \ldots \ldots \ldots \ldots \ldots$ & 54075 & 100 & 66100 & 100 \\
\hline
\end{tabular}

\footnotetext{
${ }^{1}$ D'après Anatole Kopp, L'architecture de la reconstruction en France, 1945-1953, Paris, Editions du Moniteur,
} 1982. 
ANNEXE II

CHRONOLOGIE DES TEXTES LEGISLATIFS ET REGLEMENTAIRES

\begin{tabular}{|c|c|}
\hline 1919 & $\begin{array}{l}\text { loi Cornudet (14 mars 1919): création des plans d'embellissement, d'aménagement et } \\
\text { d'extension des villes. }\end{array}$ \\
\hline 1924 & loi du 19 juillet: prolongement de la loi précédente aux lotissements. \\
\hline 1932 & loi du 14 mai: projet d'aménagement pour la région parisienne. \\
\hline 1940 & $\begin{array}{l}\text { loi du } 22 \text { août 1940: prise en charge par l'Etat des travaux de déblaiement dans les } \\
\text { localités nécessitant un plan d'urbanisme et d'aménagement pour leur reconstruction. } \\
\text { loi du constitution d'un Commissariat à la Reconstruction immobilière, assisté d'un } \\
\text { Comité national de la Reconstruction. }\end{array}$ \\
\hline 1941 & $\begin{array}{l}\text { loi du } 2 \text { février: transfert de tous les services d'urbanisme à un comité permanent } \\
\text { d'aménagement. }\end{array}$ \\
\hline 1943 & $\begin{array}{l}\text { loi du } 15 \text { mars: création des inspecteurs généraux de l'Urbanisme. } \\
\text { loi d'urbanisme du } 15 \text { juin: coordination des travaux et programmes d'équipement } \\
\text { public (définition du zoning, rôle du préfet renforcé en matière de permis de } \\
\text { construire, financement des projets des communes à l'Etat). }\end{array}$ \\
\hline 1944 & $\begin{array}{l}\text { décret du } 16 \text { novembre: création du ministère de la Reconstruction et de l'Urbanisme } \\
\text { (premier titulaire: Raoul Dautry). }\end{array}$ \\
\hline 1945 & $\begin{array}{l}\text { ordonnance du } 21 \text { avril: fixation des compétences du MRU, chargé de l'ensemble des } \\
\text { questions touchant à l'urbanisme ("établir le plan général de reconstruction"). } \\
\text { décret du } 21 \text { juin } 1945 \text { : relatif aux projets de reconstruction et d'aménagement des } \\
\text { communes sinistrées. } \\
\text { ordonnance du } 11 \text { octobre: mesures exceptionnelles et temporaires en vue de remédier } \\
\text { à la crise du logement. }\end{array}$ \\
\hline 1946 & $\begin{array}{l}\text { décret du } 3 \text { janvier: création du Commissariat général au Plan de Modernisation et } \\
\text { d'Equipement. } \\
\text { loi du } 16 \text { mai: création des associations syndicales de remembrement ou de } \\
\text { reconstruction (fonctionnement fixé par décret du } 11 \text { octobre). } \\
\text { loi sur la reconstruction du } 28 \text { octobre: inscription du principe de l'indemnisation des } \\
\text { sinistrés par la réparation des dommages subis par faits de guerre. }\end{array}$ \\
\hline 1947 & $\begin{array}{l}\text { loi du } 30 \text { mars: relative aux associations de sinistrés et au financement de la } \\
\text { reconstruction. }\end{array}$ \\
\hline 1948 & $\begin{array}{l}\text { loi du } 21 \text { mars: création de la Caisse autonome de la Reconstruction. } \\
11 \text { septembre: Eugène Claudius-Petit ministre de la Reconstruction et de l'Urbanisme. }\end{array}$ \\
\hline 1949 & $\begin{array}{l}\text { circulaire du } 8 \text { juillet: définition des conditions d'application sur les projets } \\
\text { d'aménagement. }\end{array}$ \\
\hline 1950 & $\begin{array}{l}1^{\mathrm{er}} \text { février 1950: Pour un plan national d'aménagement du territoire, MRU. } \\
2 \text { novembre: rejet par l'Assemblée nationale de la loi foncière élargissant le droit } \\
\text { d'expropriation de l'Etat (de nouveau le } 8 \text { novembre 1951). }\end{array}$ \\
\hline
\end{tabular}

